\title{
Vibrations of non-homogenous thick plate on two-parameter elastic foundation subjected to moving oscillator
}

\author{
Magdalena Ataman ${ }^{1, *}$ \\ ${ }^{1}$ Warsaw University of Technology, Faculty of Civil Engineering, Al. Armii Ludowej 16, 00-637 \\ Warsaw, Poland
}

\begin{abstract}
In the paper the thick plate on the two-parameter Winkler foundation subjected to the one-mass oscillator is analysed. The oscillator is moving at uniform speed, parallel to the edge of the rectangular plate. The foundation is defined by two elastic constants $k_{z}$ and $k_{t}$. Equations of motion of the analysed system are submitted and solved. The paper includes numerical example.
\end{abstract}

\section{Introduction}

Equations of motion of thick plate can be obtained by a few means [1-2]. The momentum conservation law and the angular momentum conservation law in the case of an infinitesimal element of plate are determined by a resultant vector and a resultant moment. Simultaneous equation them to zero provides to equations of motion. The rule of virtual work (d'Alembert's rule), when forces and moments of inertia are taken into consideration, as well as the Hamilton rule are the most frequent used during derivation of equations of motion of plate. Particular attention should be paid to the Hamilton's rule, which allows to obtain, apart from equations of motion, natural kinematic, static and mixed boundary conditions. The variation rule is very important as a basis for computer methods like FEM used to static and dynamics computations. Dynamic equations of motion of thick plate were derived by Reissner [3], Vlasov [4], Mindlin [5], Ambarcumian [6] in 50's of last century and later by many others, e.g. [7-10]. Because of vertical and horizontal reactions of soil there are a shield-plate coupled effects in thick plate supported by three-parameter Winkler foundation. In general, these effects provide to tenth order partial differential equation of motion of analysed plate. In this paper an assumption concerning separation of shield and plate effects will be made. In spite of this assumption the obtained equation of motion is complicated when we want to solve it using analytical method.

* Corresponding author: m.ataman@il.pw.edu.pl 


\section{Governing equations of the problem}

Assuming model of elastic Winkler foundation described by two parameters (Fig. 1), components of soil reaction can be written:

$$
p_{z}=k_{z} w, \quad p_{t}=k_{t} u_{i}, \quad i=x, y .
$$

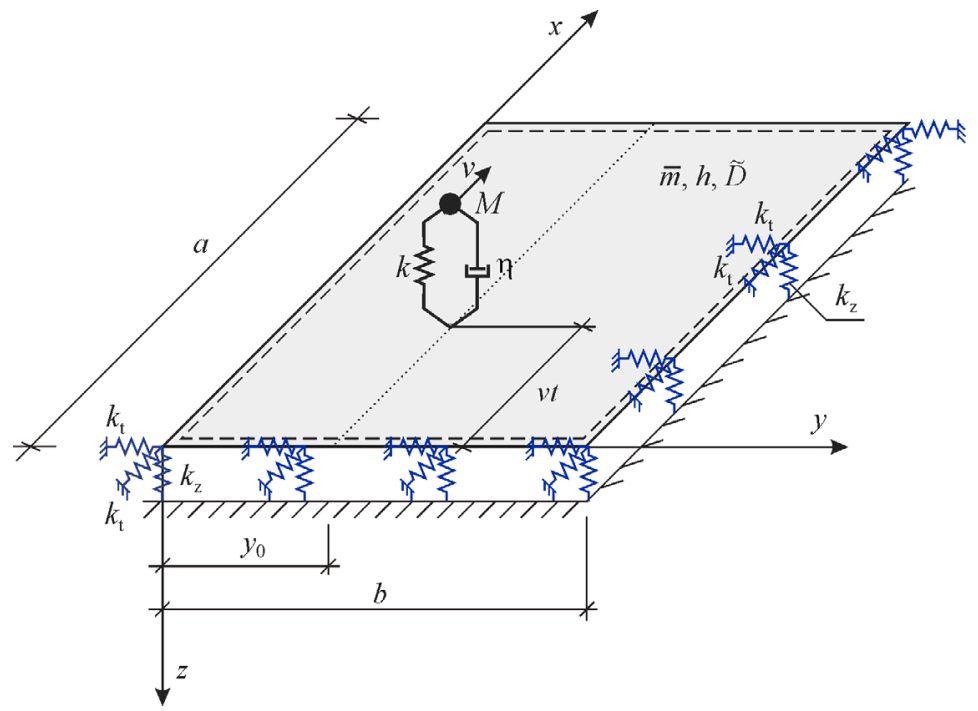

Fig. 1. Thick plate resting on generalised three-directional Winkler foundation subject to moving onemass oscillator.

Tangential and normal forces of soil reaction at the bottom of plate are expressed:

$$
X_{1}=\frac{k_{t} h}{4} \frac{\partial w}{\partial x}+\frac{k_{t} h^{3}}{24(1-v)} \chi_{x}, \quad Y_{1}=\frac{k_{t} h}{4} \frac{\partial w}{\partial y}+\frac{k_{t} h^{3}}{24(1-v)} \chi_{y}, \quad Z_{2}=p-k_{z} w
$$

Basic equation of motion of the thick plate is following [2]:

$$
\begin{gathered}
\nabla^{4} w+\frac{\rho h}{D} \frac{\partial^{2} w}{\partial t^{2}}-\frac{17-6 v}{60 D(1-v)} \rho h^{3} \nabla^{2}\left(\frac{\partial^{2} w}{\partial t^{2}}\right)+\frac{\rho^{2} h^{6}}{60 D^{2}(1-v)} \frac{\partial^{4} w}{\partial t^{4}}+ \\
+k_{z}\left[\frac{w}{D}-\frac{(2-v) h^{2}}{10 D(1-v)} \nabla^{2} w+\frac{\rho h^{5}}{60 D^{2}(1-v)} \frac{\partial^{2} w}{\partial t^{2}}\right]+ \\
-\frac{1}{1-\mu}\left\{\left[\frac{k_{t} h^{2}}{4} \nabla^{2}-\mu\left(\rho h \frac{\partial^{2}}{\partial t^{2}}+k_{z}\right)\right]\left[\frac{w}{D}-\frac{(2-v) h^{2}}{60 D(1-v)} \nabla^{2} w+\frac{\rho h^{5}}{360 D^{2}(1-v)} \frac{\partial^{2} w}{\partial t^{2}}\right]\right\}= \\
=\frac{p}{D}-\frac{(2-v) h^{2}}{10 D(1-v)} \nabla^{2} p+\frac{\rho h^{5}}{60 D^{2}(1-v)} \frac{\partial^{2} p}{\partial t^{2}}+ \\
+\frac{\mu}{1+\mu}\left[\frac{p}{D}+-\frac{(2-v) h^{2}}{60 D(1-v)} \nabla^{2} p+\frac{\rho h^{5}}{360 D^{2}(1-v)} \frac{\partial^{2} p}{\partial t^{2}}\right]
\end{gathered}
$$


where $\mu=\frac{k_{t} h^{4}}{24 D(1-v)}$ is the dimensionless factor describing influence of unknown functions $\chi_{x}$ i $\chi_{y}$ to the vertical forces at the bottom of the plate, $D=\frac{E h^{3}}{12\left(1-v^{2}\right)}$ is the flexural rigidity of the plate, $w$ is the plate deflection, $\rho$ is the density of the plate, $h$ is the plate thickness, and $p$ is the external load per unit area normal to the top surface. It should be mentioned that for factors $k_{t}$ describing actual subgrades $\mu$ factor is considerably smaller than 1 . When $\mu=0$, the form of equation of motion (3) is simpler and can be written as follows:

$$
\begin{gathered}
\nabla^{4} w+\frac{\rho h}{D} \frac{\partial^{2} w}{\partial t^{2}}-\frac{17-6 v}{60 D(1-v)} \rho h^{3} \nabla^{2}\left(\frac{\partial^{2} w}{\partial t^{2}}\right)+\frac{\rho^{2} h^{6}}{60 D^{2}(1-v)} \frac{\partial^{4} w}{\partial t^{4}}+ \\
+k_{z}\left[\frac{w}{D}-\frac{(2-v) h^{2}}{10 D(1-v)} \nabla^{2} w+\frac{\rho h^{5}}{60 D^{2}(1-v)} \frac{\partial^{2} w}{\partial t^{2}}\right]+ \\
-\left\{\left[\frac{k_{t} h^{2}}{4} \nabla^{2}\right]\left[\frac{w}{D}-\frac{(2-v) h^{2}}{60 D(1-v)} \nabla^{2} w+\frac{\rho h^{5}}{360 D^{2}(1-v)} \frac{\partial^{2} w}{\partial t^{2}}\right]\right\} \\
=\frac{p}{D}-\frac{(2-v) h^{2}}{10 D(1-v)} \nabla^{2} p+\frac{\rho h^{5}}{60 D^{2}(1-v)} \frac{\partial^{2} p}{\partial t^{2}}
\end{gathered}
$$

The equation of motion of one-mass oscillator should be connected to the equation of motion of the plate (4) [7]

$$
M \frac{\mathrm{d}^{2} w_{1}}{\mathrm{~d} t^{2}}+\eta\left(\frac{\mathrm{d} w_{1}}{\mathrm{~d} t}-\frac{\mathrm{d} w}{\mathrm{~d} t}\right)+k\left(w_{1}-w\right)=0, \quad \frac{\mathrm{d} w(v t, t)}{\mathrm{d} t}=\left.\frac{\partial w}{\partial t}\right|_{x=v t}+\left.v \frac{\partial w}{\partial x}\right|_{x=v t} .
$$

where: $\quad M$ is the mass of the oscillator,

$k$ is the elastic constant of the oscillator,

$\eta$ is the damping coefficient of the oscillator,

$w_{1}$ is the vertical displacement of the oscillator mass.

Dynamic pressure of oscillator acting to the plate describes formula:

$$
N(t)=M g-M \frac{\mathrm{d}^{2} w_{1}}{\mathrm{~d} t^{2}}
$$

Moving load can be written as follows:

$$
p=p(x, y, t)=N(t) \delta(x-v t) \delta\left(y-y_{0}\right)=\left(M g-M \frac{\mathrm{d}^{2} w_{1}}{\mathrm{~d} t^{2}}\right) \delta(x-v t) \delta\left(y-y_{0}\right) .
$$

When $P=M g$ is introduced into equation (7), load and its derivatives can be expressed: 


$$
\begin{gathered}
p=p(x, y, t)=p_{1}(x, y, t)+p_{2}(x, y, t) \\
p(x, y, t)=\left(P-M \frac{\mathrm{d}^{2} \mathrm{w}_{1}}{\mathrm{~d} t^{2}}\right) \delta(x-v t) \delta\left(y-y_{0}\right)=\sum_{n} \sum_{m} f_{n, m}(t) \sin \alpha_{m} x \sin \beta_{n} y, \\
f_{n, m}(t)=\frac{4}{a b}\left(P-M \frac{\mathrm{d}^{2} \mathrm{w}_{1}}{\mathrm{~d} \mathrm{t}^{2}}\right) \sin \alpha_{m} v t \sin \beta_{n} y_{0}, \\
\nabla^{2} p(x, y, t)= \\
=\frac{4}{a b}\left(P-M \frac{\mathrm{d}^{2} \mathrm{w}_{1}}{\mathrm{~d} t^{2}}\right) \sum_{n} \sum_{m}\left(\alpha_{m}{ }^{2}+\beta_{n}{ }^{2}\right) \sin \alpha_{m} v t \sin \beta_{n} y_{0} \sin \alpha_{m} x \sin \beta_{n} y, \\
=-\frac{\partial^{2} p(x, y, t)}{a b}= \\
-\frac{4}{a b} M \frac{\mathrm{d}^{4} t^{2}}{\mathrm{~d} t^{4}} \sum_{n} \sum_{m} \sin \alpha_{m} v t \sin \beta_{n} y_{0} \sin \alpha_{m} x \sin \beta_{n} y .
\end{gathered}
$$

To obtain solution of the problem a system of two differential equations (4) and (5) should be solved. We assume the elastic plate is rectangular of $a$ and $b$ sides and $h$ thickness. All sides of the plate are simply supported therefore the deflected form is represented by the double series

$$
w=w(x, y, t)=\sum_{n=1}^{\infty} \sum_{m=1}^{\infty} q_{n, m}(t) \sin \alpha_{n} x \sin \beta_{m} y, \quad \alpha_{n}=\frac{n \pi}{a}, \beta_{m}=\frac{m \pi}{b} .
$$

Substitution (9) in (4) and (5) for $n=m=1$ Bubnov-Galerkin method in (4) yields the system of two coupled differential equations of sixth order depending on time:

$$
\begin{gathered}
M \frac{\mathrm{d}^{2} w_{1}}{\mathrm{~d} t^{2}}+\eta \frac{\mathrm{d} w_{1}}{\mathrm{~d} t}+k w_{1}= \\
=(\eta \dot{q}+k q) \sin \frac{\pi v t}{a} \sin \frac{\pi y_{0}}{b}+\eta q \frac{\pi v}{a} \cos \frac{\pi v t}{a} \sin \frac{\pi y_{0}}{b}, \\
q^{I V}+\frac{1}{12}\left(\frac{c_{p}}{h}\right)^{2} A \ddot{q}+\frac{1}{576}\left(\frac{c_{p}}{h}\right)^{4} Q^{2} q=\left\{\frac{2 c_{p}^{2}}{\rho h^{3} a b}\left(P-M \frac{\mathrm{d}^{2} \mathrm{w}_{1}}{\mathrm{~d} t^{2}}\right)[10(1-v)+\right. \\
\left.\left.+\tilde{\gamma}^{2}(2-v)-\frac{\rho h^{5} \alpha^{2} v^{2}}{12 D}\right]-\frac{4 M}{\rho h a b} \frac{\mathrm{d}^{4} w_{1}}{\mathrm{~d} t^{4}}\right\} \sin \frac{\pi v t}{a} \sin \frac{\pi y_{0}}{b},
\end{gathered}
$$

where: $\quad \theta=\frac{k_{z}}{k_{t}}, \quad \mu=\frac{k_{t} h^{4}}{24 D(1-v)}, \quad \mu_{z}=\frac{k_{z} h^{4}}{D}, \quad \tilde{\gamma}=h^{2}\left(\alpha^{2}+\beta^{2}\right), \quad \lambda=\frac{a}{b}$,

$$
\begin{aligned}
& \xi=\frac{h}{a}, \quad \gamma=\pi \xi^{2}\left(1+\lambda^{2}\right), \quad c_{p}^{2}=\frac{E}{\rho\left(1-v^{2}\right)}, \\
& A=60(1-v)+(17-6 v) \tilde{\gamma}^{2}+\mu_{z}\left(1+\frac{\theta \tilde{\gamma}^{2}}{24}\right),
\end{aligned}
$$




$$
\begin{aligned}
& \hat{\gamma}^{4}=\tilde{\gamma}^{4}+\mu_{z}\left[1+\frac{2-v}{10(1-v)} \tilde{\gamma}^{2}+\frac{\theta \tilde{\gamma}^{2}}{4(1-v)}\left(1+\frac{2-v}{60(1-v)} \tilde{\gamma}^{2}\right)\right], \\
& Q=4 \hat{\gamma}^{2}[15(1-v)] .
\end{aligned}
$$

The above system of linear equations is solved numerically using Mathematica code. Equations (10) are valid for $t \in\left\langle 0 ; \frac{a}{v}\right\rangle$. When $t \geq \frac{a}{v}$, the free vibrations of plate and oscillator are analysed. Assuming the oscillator is moving on the rigid surface, equations of motion of oscillator and plate are given by two uncoupled equations:

$$
M \frac{\mathrm{d}^{2} \widetilde{w}_{1}}{\mathrm{dt}^{2}}+\eta \frac{\mathrm{d} \widetilde{w}_{1}}{\mathrm{dt}}+k \widetilde{w}_{1}=0, \quad \widetilde{q}^{I V}+\frac{1}{12}\left(\frac{c_{p}}{h}\right)^{2} A \ddot{\widetilde{q}}+\frac{1}{576}\left(\frac{c_{p}}{h}\right)^{4} Q^{2} \widetilde{q}=0 .
$$

Substitution of dimensionless eigenvalues of plate yields:

$$
\begin{gathered}
\omega^{4}-\frac{1}{12}\left(\frac{c_{p}}{h}\right)^{2} A \omega^{2}+\frac{1}{576}\left(\frac{c_{p}}{h}\right)^{4} Q^{2}=0 \rightarrow \Omega_{1 / 3}=\frac{1}{\tilde{\gamma}^{2}}\left\{\frac{1}{2}\left[P \mp\left(P^{2}-Q^{2}\right)^{4}\right]\right\}^{1 / 2}, \\
\Omega_{1}=\frac{\omega_{1}}{\omega_{k l}}, \quad \Omega_{3}=\frac{\omega_{3}}{\omega_{k l}}, \quad \omega_{k l}=\pi^{2}\left(\frac{m^{2}}{a^{2}}+\frac{n^{2}}{b^{2}}\right)\left(\frac{D}{\rho h}\right)^{1 / 2}=\frac{\tilde{\gamma}_{m, n}{ }^{2} c_{p}}{h \sqrt{12}}, \quad m=n=1 .
\end{gathered}
$$

Natural frequency of oscillator is given by expression $\omega_{\text {osc }}=\sqrt{\frac{k}{M}}$.

Solution of homogenous differential equations of motion of plate and oscillator is given by:

$$
\begin{gathered}
\widetilde{w}_{1}=A_{1} \cos \omega_{o s c} t+A_{2} \sin \omega_{\text {osc }} t, \\
\widetilde{q}=C_{1} \cos \omega_{1} t+C_{2} \sin \omega_{1} t+C_{3} \cos \omega_{3} t+C_{4} \sin \omega_{3} t .
\end{gathered}
$$

The constants $A_{1}, A_{2}$ and $C_{1} \div C_{4}$ are to be determined from the initial conditions for $t=\frac{a}{v}$. Introduction of notations $\widetilde{w}_{1}$ and $\widetilde{w}$ instead of $w_{1}$ and $w$ for $t \geq \frac{a}{v}$ gives following expressions:

$$
\begin{gathered}
t=\frac{a}{v} \rightarrow w_{1}=\widetilde{w}_{1}, \quad \frac{\mathrm{d} w_{1}}{\mathrm{~d} t}=\frac{\mathrm{d} \widetilde{w}_{1}}{\mathrm{~d} t}, \quad \frac{\mathrm{d}^{2} w_{1}}{\mathrm{~d} t^{2}}=\frac{\mathrm{d}^{2} \widetilde{w}_{1}}{\mathrm{~d} t^{2}}, \quad \frac{\mathrm{d}^{3} w_{1}}{\mathrm{~d} t^{3}}=\frac{\mathrm{d}^{3} \widetilde{w}_{1}}{\mathrm{~d} t^{3}}, \\
w=\widetilde{w}, \quad \frac{\mathrm{d} w}{\mathrm{~d} t}=\frac{\mathrm{d} \widetilde{w}}{\mathrm{~d} t}, \quad \frac{\mathrm{d}^{2} w}{\mathrm{~d} t^{2}}=\frac{\mathrm{d}^{2} \widetilde{w}}{\mathrm{~d} t^{2}}, \quad \frac{\mathrm{d}^{3} w}{\mathrm{~d} t^{3}}=\frac{\mathrm{d}^{3} \widetilde{w}}{\mathrm{~d} t^{3}} .
\end{gathered}
$$

\section{Transversally non-homogenous plate}

When consider plate of modulus of elasticity $E(z)$, and uniform Poisson's ratio $v$, the flexural rigidity of the plate in Eq. (3) and (4) is given by formula: 


$$
D=\tilde{D}=\int_{-\frac{h}{2}-e}^{\frac{h}{2}-e} \frac{E(\tilde{z}) \tilde{z}^{2}}{1-v^{2}} d \tilde{z}, \quad e=\frac{\int_{\frac{h}{2}}^{\frac{h}{2}} E(z) z d z}{\int_{-\frac{h}{2}}^{\frac{h}{2}} E(z) d z}
$$

where $e$ is a distance for which shield and plate effects are separated.

For the three-layered plate $e$ can be found using expression:

$$
e=\frac{E_{1} \int_{-\frac{h}{2}}^{-\frac{h}{2}+h_{1}} z d z+E_{2} \int_{-\frac{h}{2}+h_{1}}^{-\frac{h}{2}+h_{1}+h_{2}} z d z+E_{3} \int_{-\frac{h}{2}+h_{1}+h_{2}}^{-\frac{h}{2}+h} z d z}{E_{1} \int_{-\frac{h}{2}}^{-\frac{h}{2}+h_{1}} d z+E_{2} \int_{-\frac{h}{2}+h_{1}}^{-\frac{h}{2}+h_{1}+h_{2}} d z+E_{3} \int_{-\frac{h}{2}+h_{1}+h_{2}}^{-\frac{h}{2}+h} d z},
$$

where $E_{i}$ and $h_{i}$ are modulus of elasticity and thickness of $i$-th layer respectively.

Displacements and internal forces are referred to Oxy - the equivalent neutral plane of the plate.

\section{Numerical example}

The system of two differential equations of motion of oscillator and plate presented in the paper is solved using MATHEMATICA code. Dimensionless coordinates are introduced into calculations. Two cases of vibrations are investigated in the paper. The first case when the oscillator is moving on the plate $\zeta=\frac{v t}{a} \leq 1$, and the second one after the oscillator descending the plate $\zeta \geq 1$. According to the order of the system of differential equations of motion eight initial conditions equal to zero are assumed. The power of double series describing plate deflections is one.

In numerical example influence of linear viscous damping in the oscillator and in the plate was taken into account. Proportionality of the damping coefficient $c_{e}$ to the mass of the plate $\bar{m}$, and proportionality of the damping coefficient $c_{i}$ to Young's modulus $E$ were assumed and the substitutional damping number $\xi_{n}$ was defined:

$$
c_{e}=\alpha_{e} \bar{m}, \quad c_{i}=\alpha_{i} E, \quad \xi_{n}=\frac{1}{2}\left(\frac{\alpha_{e}}{\omega_{1}}+\alpha_{i} \omega_{1}\right) \text {. }
$$

Above assumptions change the equation of motion of the plate and were taken into account in numerical example.

We consider a square plate of sides $a=b=1 \mathrm{~m}$ and thickness $h=0.2 \mathrm{~m}$. Following parameters for plate material are assumed: Young's modulus is $E=20 \cdot 10^{9} \mathrm{~Pa}$, Poisson's ratio is $v=0.167$, density is $\rho=2200 \mathrm{~kg} / \mathrm{m}^{3}$, viscosity coefficient is $\eta_{1}=90000 \mathrm{Ns} / \mathrm{m}$, 
modulus of elastic subgrade reaction in vertical direction is $k_{z}=50 \cdot 10^{6} \mathrm{~N} / \mathrm{m}^{3}$ and in horizontal directions $k_{t}=0,5 k_{z}$. Oscillator's mass is $M=6000 \mathrm{~kg}$, its elastic constant is $k=16 \cdot 10^{5} \mathrm{~N} / \mathrm{m}$ and damping coefficient is $\eta=14000 \mathrm{~kg} / \mathrm{s}$. The oscillator is moving parallel to $x$ axis at uniform speed. Using Mathematica code dynamic deflection of the plate midpoint and deflection of the mass of the oscillator were determined and analysed for various values of oscillator velocity. In the figures dynamic case $v=100 \mathrm{~m} / \mathrm{s}$ and static case $v=0.1 \mathrm{~m} / \mathrm{s}$, for verification purpose, are presented. Figures present deflection of the plate and the oscillator for forced and free vibrations.

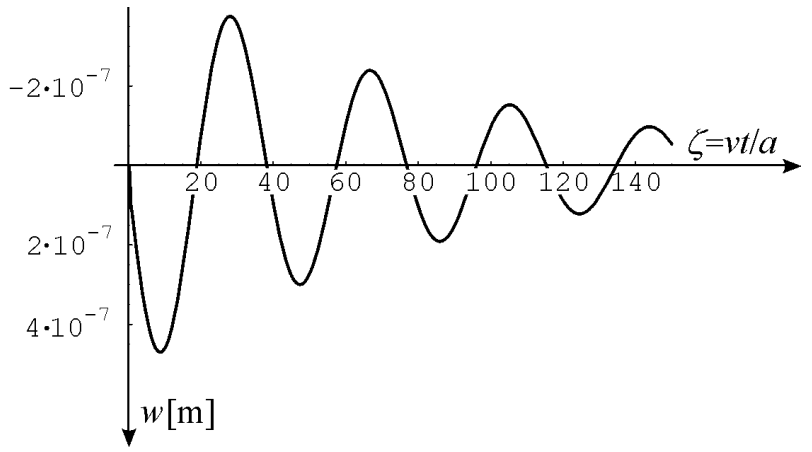

Fig. 2. Deflection of viscoelastic oscillator, velocity $v=100 \mathrm{~m} / \mathrm{s}$.

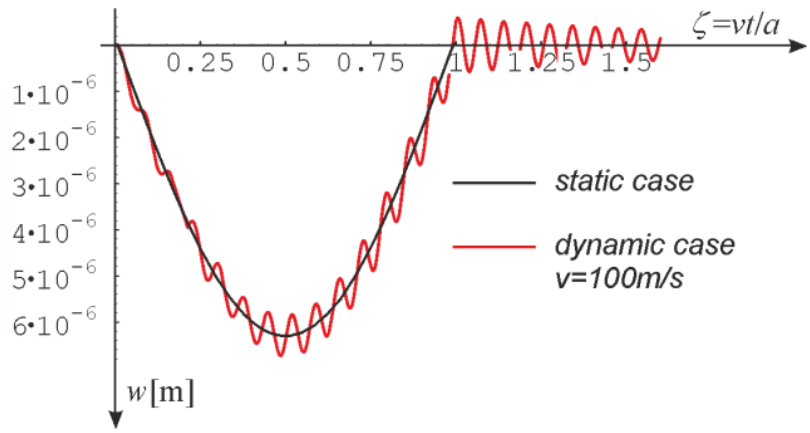

Fig. 3. Deflection of thick plate midpoint. Dynamic case $v=100 \mathrm{~m} / \mathrm{s}$.

\section{Conclusions}

In the paper theoretical basis of the thick plate on the generalised Winkler foundation under one-mass oscillator are submitted. The equations of motion of the analysed oscillator and the plate are of second and fourth order in respect to time. The instant when the oscillator is descending the plate requires further investigations because the oscillator impact in the plate support causes disturbance in the movement.

\section{References}

1. W. Szcześniak, Wybrane zagadnienia z dynamiki płyt, OWPW, Warszawa (2000) 
2. W. Szcześniak, Eng. Trans., IPPT PAN, 35, 3, 347-374 (1987)

3. E. Reissner, J. Appl. Mech., 25, 1 (1958)

4. B.F. Vlasov, Wiestnik MGSU, 2 (1957)

5. R.D. Mindlin, J. Appl. Mech. 18, 1, 31-38 (1951)

6. S.A. Ambarcumian, Theory of anisotropic plates, Nauka, Moskwa (1987)

7. W. Szcześniak, Eng. Trans., IPPT PAN, 20, 3, 455-478 (1972)

8. W. Szcześniak, ACE, 22, 1, 107-127 (1976)

9. W. Szcześniak, Eng. Trans., IPPT PAN, 3, 1-2, 37-53 (1985)

10. W. Szcześniak, Eng. Trans., IPPT PAN, 37, 1, 87-115 (1989) 